\title{
Calculating the effective thermal conductivity of gray cast iron by using an interconnected graphite model
}

\author{
Guang-hua Wang', *Yan-xiang Li ${ }^{1,2}$ \\ 1. School of Materials Science and Engineering, Tsinghua University, Beijing 100084, China \\ 2. Key Laboratory for Advanced Materials Processing Technology, Ministry of Education, Beijing 100084, China
}

\begin{abstract}
A new theoretical model of gray cast iron taking into account a locally interconnected structure of flake graphite was designed, and the corresponding effective thermal conductivity was calculated using the thermal resistance network method. The calculated results are obviously higher than that of the effective medium approximation assuming that graphite is distributed in isolation. It is suggested that the interconnected structure significantly enhances the overall thermal conductivity. Moreover, it is shown that high anisotropy of graphite thermal conductivity, high volume fraction of graphite, and small aspect ratio of flake graphite will cause the connectivity effects of graphite to more obviously improve the overall thermal conductivity. Higher graphite volume fraction, lower aspect ratio and higher matrix thermal conductivity are beneficial to obtain a high thermal conductivity gray cast iron. This work can provide guidance and reference for the development of high thermal conductivity gray cast iron and the design of high thermal conductivity composites with similar locally interconnected structures.
\end{abstract}

Key words: gray cast iron; effective thermal conductivity; flake graphite; interconnected graphite structure CLC numbers: TG143.2 Document code:A Article ID: 1672-6421(2020)03-183-07

\begin{abstract}
Gray cast iron (GCI) is the most widely used $U_{\text {casting metallic material due to its good }}$ mechanical properties, excellent casting ability and low cost ${ }^{[1]}$. These properties in combination with high thermal conductivity allow GCI to be used in important applications in high temperature situation, such as brake disc, engine block, cylinder head, etc. The thermal conductivity is an extremely important performance index for GCI. The high thermal conductivity can help to transfer heat rapidly and prevent thermo-mechanical fatigue, deformation and hot cracking, thereby improving the service life of products ${ }^{[2]}$. To date, more and more attention has been paid to the design and development of high thermal conductivity GCI.
\end{abstract}

\footnotetext{
*Yan-xiang Li

Male, born in 1962, Ph.D., Professor. His research interests are mainly focused on casting alloys, solidification process, especially directional solidification of alloys; porous metals (aluminium foams and lotus-type metals). He has published over two hundred papers in international and domestic journals, and dozens of conference reports. He is now the Chairman of the WFO Ferrous Metals Commission and a member of the Foundry Institution of Chinese Mechanical Engineering Society.

E-mail: yanxiang@tsinghua.edu.cn
}

Received: 2020-03-02; Accepted: 2020-04-15
The effective thermal conductivity of GCI depends not only on the thermal conductivity of flake graphite and matrix, but also the volume fraction, size, shape and orientation of graphite ${ }^{[2,3]}$. Effects of these factors on the thermal conductivity of GCI are complex. It is difficult to control a single variable and timeconsuming to study the influence of these factors through experiments. So, theoretical methods are more preferred owing to their strong predictive power, low cost and reasonable accuracy, especially when the microstructure can be simplified. These methods are also widely used in the prediction of thermal conductivity of composite materials ${ }^{[4]}$. GCI can be regarded as an in-situ composite made up of matrix and flake graphite, the matrix includes ferrite, pearlite, cementite and so on. Therefore, the theoretical approaches for the thermal conductivity of composites can be used to study the thermal conductivity of GCI.

The theoretical approaches mainly include the effective medium approximation (EMA) and unit cell model ${ }^{[5]}$. EMA was first proposed by Maxwell based on the electric potential satisfying the Laplace equation to calculate the effective electrical resistivity of spherical particle composites ${ }^{[6]}$. Eucken ${ }^{[7]}$ developed Maxwell's model to describe the thermal conductivity of particle 
reinforced composites with a low volume fraction $(f \leq 10 \%)$ by setting to analog the electric potential field with the temperature field. Bruggeman ${ }^{[8]}$ established the model for composites with higher volume fractions and considered the interaction between particles by using the integral embedding principle. Hamilton et al. ${ }^{[9]}$ and Hatta et al. ${ }^{[10]}$ introduced the shape factor to generalize the Maxwell model, making it suitable for various shapes of composites. The interface thermal resistance (ITR) found by Kapitza et al. ${ }^{[11]}$ has a certain influence on the thermal conductivity of composites, especially when the size of the reinforcement is micro-nano scale. Hasselman et al. ${ }^{[12]}$ introduced the ITR and modified the Maxwell model. The prediction accuracy of this model is improved significantly when the size of the reinforcement is very small. Nan et al. ${ }^{[13]}$ established a more general EMA model considering the influence of ITR, shape size and orientation distribution. In recent years, with the development of EMA, the theory has been applied to wider areas, such as higher volume fraction ${ }^{[14]}$, multiphase ${ }^{[3,15]}$, micro-nano scale ${ }^{[16]}$, etc.

Unit cell model applies a representative volume element (RVE) composed of phases with different thermal conductivities, and the thermal conductivity of RVE is calculated according to the thermal resistance network method ${ }^{[17]}$. This model is also widely used in numerical simulation ${ }^{[18,19]}$. Zehner et al. ${ }^{[20]}$ calculated the thermal conductivity of the point-contacted spherical particle reinforced composites by constructing a cylindrical unit cell. Hsu et al. ${ }^{[21]}$ developed lumped-parameter models of 2D and 3D spatially periodic media by building rectangular and cube cells, respectively. Due to the interaction between particles considered in the model, it is more consistent with experimental results at high volume fraction than Maxwell's model ${ }^{[5]}$. Yu et al. ${ }^{[22]}$ established a model of composite thermal conductivity with embedded fractal-like tree networks based on the thermal resistance network method. Based on the integral average and resistance network method, Qian et al. ${ }^{[23]}$ calculated the effective thermal conductivity of the unit cell for spherical particle composites. The theoretical model reaches a good agreement with available numerical and experimental data. Compared with the EMA, the unit cell model is more suitable for composites with complex structural features.

For GCI, Helsing et al. ${ }^{[24]}$ established the thermal conductivity model of GCI assuming that flake graphite is distributed in isolation based on EMA. This model has been applied to the study of GCI thermal conductivity ${ }^{[25-27]}$. However, in fact, the flake graphite in each eutectic cell of GCI is interconnected ${ }^{[2,3]}$. To date, the effect of graphite connectivity on the thermal conductivity of GCI is still not clear. For composites, some studies show that the calculated results of existing theoretical models, assuming that the reinforcement is distributed in the isolated form, are always lower than that of experimental results ${ }^{[28,29]}$. In other words, the interconnected structure of reinforcement particles is helpful to improve the overall thermal conductivity. Therefore, it is very important to study the influence of this factor on the thermal conductivity of GCI.
In this study, a theoretical model of GCI with interconnected graphite structure was designed, and the corresponding effective thermal conductivity was calculated based on the unit cell model, which considers both the connectivity effect and anisotropy of thermal conductivity for graphite. This work can provide theoretical guidance and reference for the design and development of high thermal conductivity GCI.

\section{Theoretical models}

\subsection{Nan's model}

Gray cast iron is a kind of cast iron with carbon precipitated in the form of flake graphite. Therefore, it can be regarded as an in-situ composite made up of the matrix and flake graphite. The matrix includes ferrite, pearlite, cementite, and so on. For the composites with flake reinforcement, one effective method for the prediction of effective thermal conductivity is the model proposed by Nan et al. ${ }^{[13,30]}$ based on average $t$-matrix approximation (ATA). For GCI, it is considered that flake graphite is of disc shape with diameter of $l$ and thickness of $h$, and distributed in random and isolated in a continuous matrix. Its physical model is shown in Fig. 1. The corresponding mathematical model [Eqs. (1) - (5)] is shown below:

$$
\begin{gathered}
k_{\mathrm{e}}=k_{\mathrm{m}} \frac{3+f\left[2 \beta_{1}\left(1-L_{1}\right)+\beta_{2}\left(1-L_{2}\right)\right]}{3-f\left[2 \beta_{1} L_{1}+\beta_{2} L_{2}\right]} \\
\beta_{1}=\frac{k_{\mathrm{a}}-k_{\mathrm{m}}}{k_{\mathrm{m}}+L_{1}\left(k_{\mathrm{a}}-k_{\mathrm{m}}\right)} \\
\beta_{2}=\frac{k_{\mathrm{c}}-k_{\mathrm{m}}}{k_{\mathrm{m}}+L_{2}\left(k_{\mathrm{c}}-k_{\mathrm{m}}\right)} \\
L_{1}=\frac{\pi}{4} p \\
L_{2}=1-\frac{\pi}{2} p
\end{gathered}
$$

where $k_{\mathrm{e}}$ is the effective thermal conductivity of GCI, $k_{\mathrm{m}}$ is the thermal conductivity of matrix, $k_{\mathrm{a}}$ is the thermal conductivity of graphite along the flake, $k_{\mathrm{c}}$ is the thermal conductivity of graphite perpendicular to the flake, $f$ is the volume fraction of graphite, $p$ is the aspect ratio of graphite $(p=h / l), L_{i}$ is

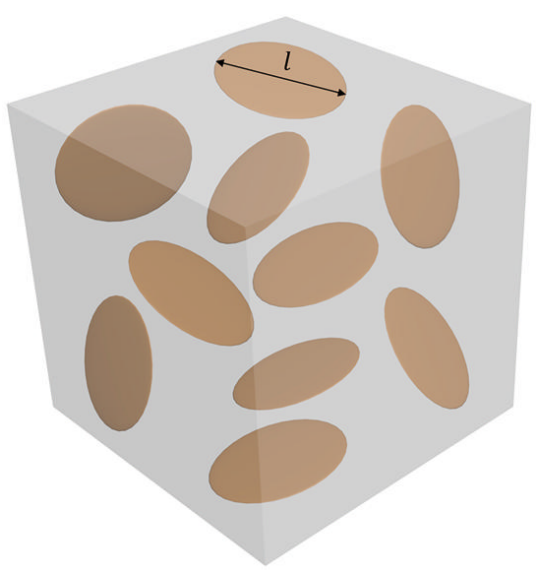

Fig. 1: Schematics of Nan's model 
geometrical factor of the disc. Compared with Helsing's model, the mathematical expression of this model is explicit, and the calculation is relatively simple.

\subsection{New model}

Industrial GCI is generally of hypoeutectic composition, the structure after eutectic solidification is mainly composed of the eutectic cell and primary austenite. The eutectic cell is composed of the flake graphite and eutectic austenite formed in the eutectic reaction stage of GCI. For the eutectic reaction process, graphite is the high melting point phase, so the graphite will firstly nucleate from the molten iron and grow into a sphere.
Then, graphite gradually branches, and the eutectic austenites precipitate synergistically among the branches. Finally, the eutectic cells are formed with interconnected flake graphite surrounded by eutectic austenite ${ }^{[31]}$. Based on such solidification process, it is assumed that all eutectic cells are sphere colonies with the same size and distribute randomly around primary austenites. The sketch of hypoeutectic GCI solidification structure is shown in Fig. 2(a). The RVE including one eutectic cell can be established, as shown in Fig. 2(b), considering the connectivity in each eutectic cell as three pieces of flake graphite interlaced distribution.
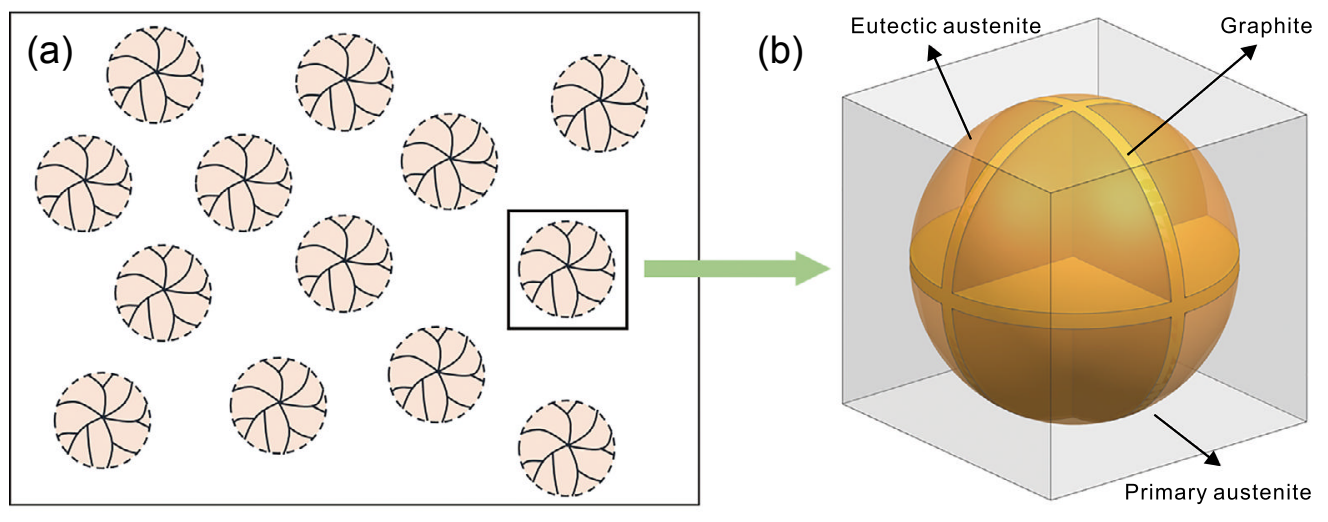

Fig. 2: Sketch of solidification structure of gray cast iron (a) and 3D unit cell of RVE (b)

Because the primary austenite and eutectic austenite are either ferrite, pearlite, or a mixture of ferrite and pearlite when cooled to room temperature, assuming that both of them are matrix with the thermal conductivity of $k_{\mathrm{m}}$, then, Fig. 2 (b) can be equivalent to the physical model shown in Fig. 3 (a). To facilitate the calculation of the effective thermal conductivity of this structure based on the thermal resistance network method, the disc-shaped flake graphite was simplified as a squared rectangle with length and width of $l$ and thickness of $h$, as shown in Fig. 3 (b). The edge length $a$ of RVE satisfies

$$
l^{3}-(l-h)^{3}=f a^{3}
$$

Then, $a=l \times \sqrt[3]{\frac{3 p-3 p^{2}+p^{3}}{f}}$. Because the geometric condition satisfies $a>l$, the aspect ratio of graphite $p$ should satisfy $3 p-3 p^{2}+p^{3}>f$. In this study, a low volume fraction of graphite is considered $(f \leq 10 \%)$, so the corresponding $p$ should be larger than 0.04 .

Due to the symmetry of thermal paths, a $1 / 8$ part of RVE is taken for calculation. It is divided into three parts as shown in Fig. 4. Then, the heat flow $\mathrm{Q}$ and its direction are given. Let $\alpha_{1}=l / a$ and $\alpha_{\mathrm{h}}=h / a$, according to the series rule, the overall effective thermal conductivity is given by

$$
k_{\mathrm{e}}^{-1}=\left(1-\alpha_{1}\right) / k_{\mathrm{m}}+\left(\alpha_{1}-\alpha_{\mathrm{h}}\right) / k_{\mathrm{p} 2}+\alpha_{\mathrm{h}} / k_{\mathrm{p} 3}
$$

(a)

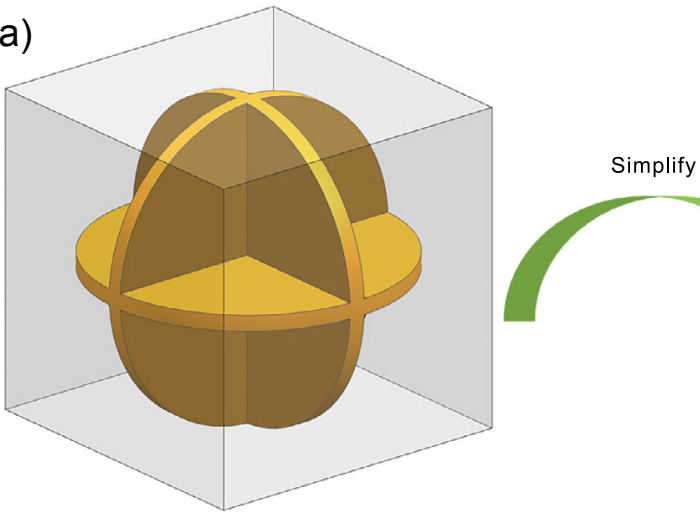

(b)

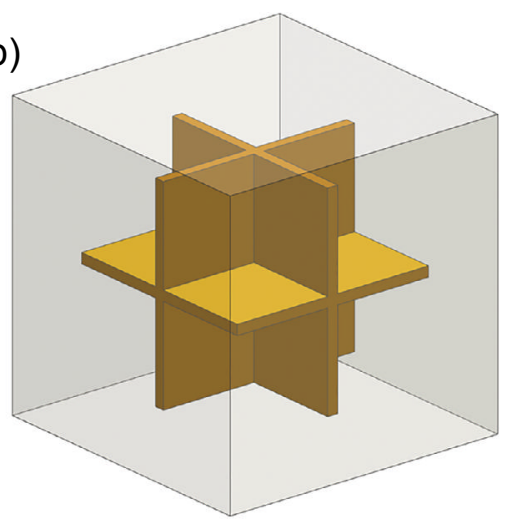

Fig. 3: 3D RVE of GCl with physical model (a) and simplified structure (b) 


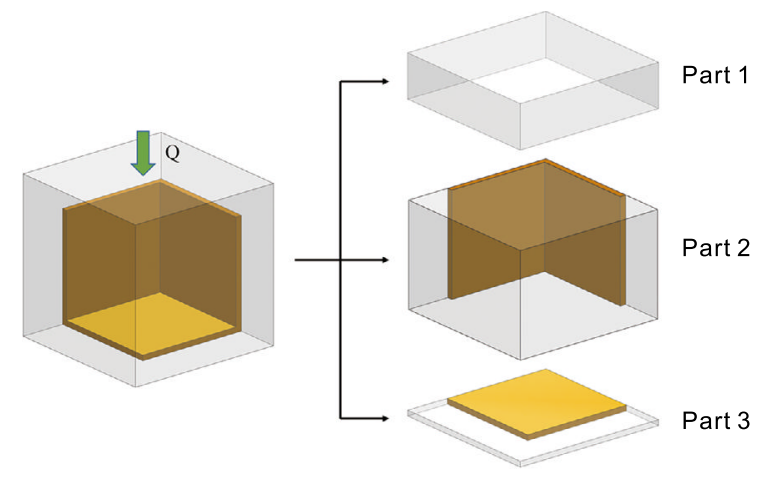

Fig. 4: Sketch of calculation process of effective thermal conductivity for a $1 / 8$ unit cell

For Part 2, any cross section was taken and divided into three parts, as shown in Fig. 5. According to the parallel rule,

$$
k_{\mathrm{p} 2}=\left(1-2 \alpha_{1} \alpha_{\mathrm{h}}+\alpha_{\mathrm{h}}^{2}\right) k_{\mathrm{m}}+\left(2 \alpha_{1} \alpha_{\mathrm{h}}-\alpha_{\mathrm{h}}^{2}\right) k_{\mathrm{a}}
$$

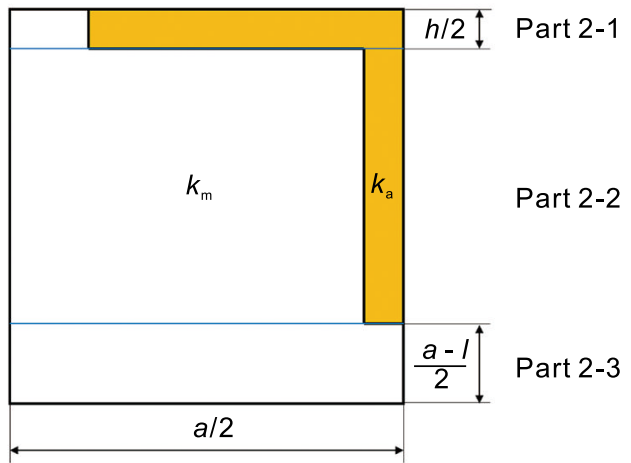

Fig. 5: Sketch of calculation process of effective thermal conductivity for Part 2

Similarly, as shown in Fig. $6, k_{\mathrm{p} 3}$ satisfies

$$
k_{\mathrm{p} 3}=\alpha_{1}\left[\left(1-\alpha_{1}\right) k_{\mathrm{m}}+\alpha_{1} k_{\mathrm{c}}\right]+\left(1-\alpha_{1}\right) k_{\mathrm{m}}
$$

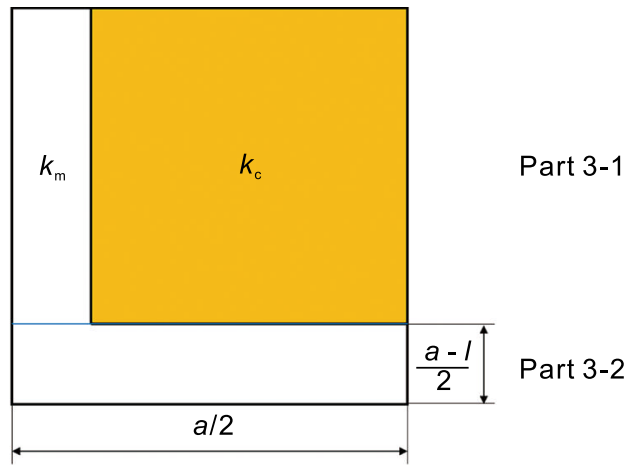

Fig. 6: Sketch of calculation process of effective thermal conductivity for Part 3

According to Eqs. (7)-(9), the effective thermal conductivity of GCI is obtained by:

$$
\begin{aligned}
k_{\mathrm{e}}^{-1}= & \frac{\left(1-\alpha_{1}\right)}{k_{\mathrm{m}}}+\frac{\left(\alpha_{1}-\alpha_{\mathrm{h}}\right)}{\left(1-2 \alpha_{1} \alpha_{\mathrm{h}}+\alpha_{\mathrm{h}}^{2}\right) k_{\mathrm{m}}+\left(2 \alpha_{1} \alpha_{\mathrm{h}}-\alpha_{\mathrm{h}}^{2}\right) k_{\mathrm{a}}} \\
& +\frac{\alpha_{\mathrm{h}}}{\left(1-\alpha_{1}^{2}\right) k_{\mathrm{m}}+\alpha_{1}^{2} k_{\mathrm{c}}}
\end{aligned}
$$

\section{Comparison between the two models}

Figure 7 shows the ratio of effective thermal conductivity to matrix thermal conductivity $\left(k_{\mathrm{e}} / k_{\mathrm{m}}\right)$ varies with the ratio of graphite thermal conductivity along the flake to matrix thermal conductivity $\left(k_{\mathrm{a}} / k_{\mathrm{m}}\right)$, where the volume fraction of graphite $f$ is $10 \%$, and the aspect ratio $p$ is 0.1 . The thermal conductivity of graphite is strong anisotropy, the ratio of graphite thermal conductivity along the flake to perpendicular to the flake $\left(k_{\mathrm{a}} / k_{\mathrm{c}}\right)$ of different types of graphite is quite different ${ }^{[32]}$. In order to study the effect of anisotropy of graphite thermal conductivity on the overall thermal conductivity, $k_{\mathrm{a}} / k_{\mathrm{c}}=20, k_{\mathrm{a}} / k_{\mathrm{c}}=100$ and $k_{\mathrm{a}} / k_{\mathrm{c}}=500$, were taken, respectively. It can be seen that the calculated results of this model are obviously higher than that of Nan's model. It is suggested that the interconnected structure of graphite significantly enhances the overall thermal conductivity. Moreover, with the increase of $k_{\mathrm{a}} / k_{\mathrm{m}}$, the effect of graphite connectivity on the overall thermal conductivity is more obvious. On the other hand, when $k_{\mathrm{a}} / k_{\mathrm{c}}$ becomes very large, the overall thermal conductivity will be diminished, and the weakened degree of our model is greater than that of Nan's model. Of course, the higher the graphitization, the higher the thermal conductivity of graphite along the flake, and the lower the thermal conductivity of graphite perpendicular to the flake ${ }^{[32,33]}$. In other words, the relationship between $k_{\mathrm{a}}$ and $k_{\mathrm{c}}$ is often negatively correlated, and the effect of $k_{\mathrm{a}}$ on overall thermal conductivity is much higher than that of $k_{\mathrm{c}}$. Therefore, with the increase of anisotropy for graphite thermal conductivity, $k_{\mathrm{a}} / k_{\mathrm{m}}$ will relatively increase, and the overall thermal conductivity will increase.

Figure 8 shows the relationship between $k_{\mathrm{e}} / k_{\mathrm{m}}$ and aspect ratio $p$, where the volume fraction of graphite $f$ is $10 \%, k_{\mathrm{a}} / k_{\mathrm{m}}=60$, and $k_{\mathrm{a}} / k_{\mathrm{c}}=200$. In the same way, the effective thermal conductivity calculated by this model is significantly higher than that calculated by Nan's model, which proves that the connectivity of graphite can form high heat transfer channel, thus improving the overall thermal conductivity. Moreover, with the increase of the aspect ratio, the overall thermal conductivity decreases monotonously. This result indicates that it is easier to obtain high thermal conductivity GCI with a low aspect ratio of flake graphite. One of the main reasons is that a low aspect ratio can expand the heat transfer channel along the flake of graphite, thus improving the overall thermal conductivity. It's worth noting that when the aspect ratio is low, the calculated results of this model are higher than that of Nan's model, compared with a high aspect ratio. Therefore, a lower aspect ratio can enhance the connectivity effect of graphite to improve the overall thermal conductivity.

In general, the aspect ratio of flake graphite is related to the size of eutectic cell, the larger the eutectic cells, the lower the aspect ratio. In other words, with the increase of eutectic size, the aspect ratio of graphite always decreases, and the effect of connectivity on the overall thermal conductivity will be improved.

Figure 9 shows the relationship between $k_{\mathrm{e}} / k_{\mathrm{m}}$ and volume fraction graphite $f$, where taking $p=0.1, k_{\mathrm{a}} / k_{\mathrm{m}}=60$, and $k_{\mathrm{a}} / k_{\mathrm{c}}=200$. With the increase of graphite volume fraction, the effective 

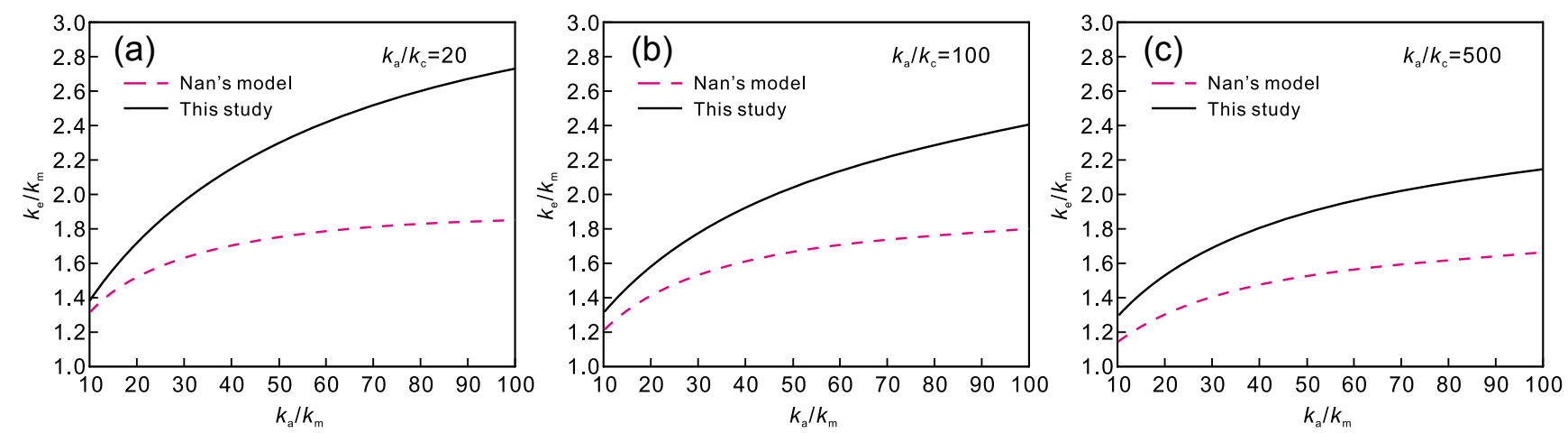

Fig. 7: Relationship between $k_{\mathrm{e}} / k_{\mathrm{m}}$ and $k_{\mathrm{a}} / k_{\mathrm{m}}$ when $f=10 \%, p=0.1$ and $k_{\mathrm{a}} / k_{\mathrm{c}}=20(\mathrm{a}), k_{\mathrm{a}} / k_{\mathrm{c}}=100(\mathrm{~b}), k_{\mathrm{a}} / k_{\mathrm{c}}=500$ (c)

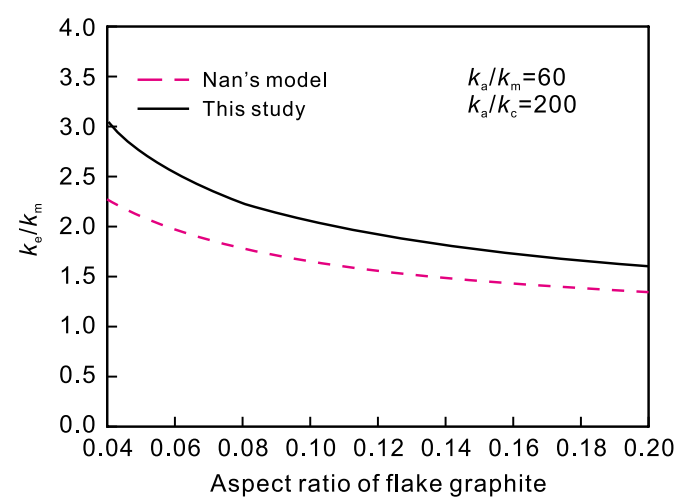

Fig. 8: Relationship between $\boldsymbol{k}_{\mathrm{e}} / \boldsymbol{k}_{\mathrm{m}}$ and aspect ratio of flake graphite when $f=10 \%, k_{\mathrm{a}} / k_{\mathrm{m}}=60, k_{\mathrm{a}} / k_{\mathrm{c}}=200$

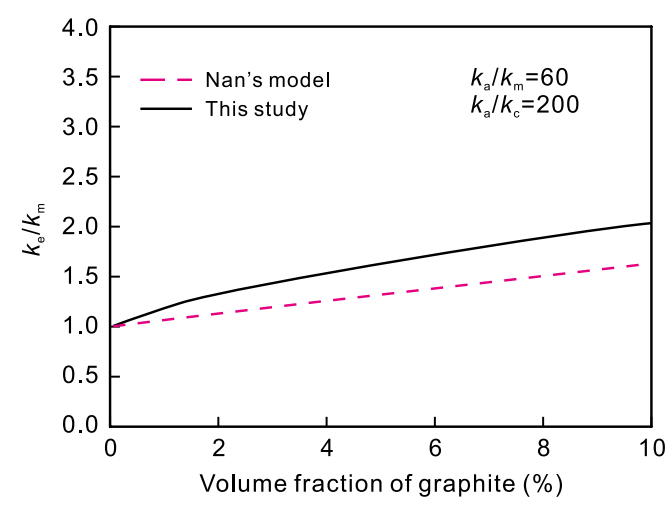

Fig. 9: Relationship between $k_{\mathrm{e}} / k_{\mathrm{m}}$ and volume fraction graphite when $p=0.1, k_{\mathrm{a}} / k_{\mathrm{m}}=60, k_{\mathrm{a}} / k_{\mathrm{c}}=200$

thermal conductivity increases monotonously. Moreover, the higher the graphite volume fraction, the higher the calculated results of this model compared with Nan's model. This result shows that with the increase of graphite volume fraction, the connectivity effects of graphite are more obvious in improving the overall thermal conductivity.

\section{Application to gray cast iron}

\subsection{Material parameters}

Table 1 gives the values of thermal conductivity of each constituent of GCI at $300 \mathrm{~K}^{[2,24]}$. The thermal conductivity of alloyed ferrite $k_{\alpha}$ at $300 \mathrm{~K}$ can be calculated by the equation the authors established earlier ${ }^{[34]}$

$$
k_{\alpha}=\frac{1}{1.61 \times 10^{-2}+\frac{\sum_{i} \rho_{i}^{\prime} c_{i}}{L_{0} T_{r}}}+\frac{1}{5.65 \times 10^{-2}+1.36 \times 10^{-2} \sum_{i} c_{i} \Gamma_{i}}
$$

where $\rho_{i}^{\prime}$ is the contribution coefficient to electrical resistivity for alloying element $i, c_{i}$ is the atomic fraction, $\Gamma_{i}$ is the impurity scattering parameter, $L_{0}=2.443 \times 10^{-8}{\mathrm{~W} \Omega \mathrm{K}^{-2}}^{-}$is the Lorentz constant, $T_{r}=300 \mathrm{~K}$ is the room temperature. Table 2 gives parameters $\rho_{i}^{\prime}$ and $\Gamma_{i}$ of some alloying elements.

Table 1: Thermal conductivity of structural constituents of cast iron at $300 \mathrm{~K}$

\begin{tabular}{|ccc|}
\hline Structural constituent & $\begin{array}{c}\text { Thermal conductivity } \\
\left(\mathbf{W} \cdot \mathbf{m}^{-1} \cdot \mathbf{K}^{-1}\right)\end{array}$ & Ref. \\
\hline $\begin{array}{c}\text { Graphite along the flake, } k_{\mathrm{a}} \\
\text { Graphite perpendicular to the } \\
\text { flake, } k_{\mathrm{c}}\end{array}$ & 1,950 & {$[2]$} \\
Alloyed ferrite, $k_{\alpha}$ & 5.7 & {$[2]$} \\
Cementite, $k_{\mathrm{v}}$ & $20-40$ & {$[2]$} \\
Pearlite, $k_{\beta}$ & 8 & {$[24]$} \\
\hline
\end{tabular}

Table 2: Experimental values for $\rho_{i}^{\prime}$ and calculated values for $\Gamma_{i}$ of some elements at $300 \mathrm{~K}^{[24,34]}$

\begin{tabular}{ccccccccc} 
& Al & Si & Cr & Mn & Ni & Mo & W & Sn \\
\hline$\rho_{i}^{\prime}\left(\mu \Omega \cdot \mathrm{cm} \cdot(\mathrm{at} \%)^{-1}\right)$ & 6.4 & 7.0 & 4.6 & 5.0 & 2.7 & 4.8 & 5.0 & 10.0 \\
$\Gamma_{i}\left(\mathrm{~m} \cdot \mathrm{K} \cdot \mathrm{W}^{-1} \cdot(\mathrm{at} \%)^{-1}\right)$ & 0.1 & 0.6 & 0 & 0 & 0 & 1.7 & 8.5 & 12.0
\end{tabular}

The effective thermal conductivity of pearlite is calculated according to Helsing's equation ${ }^{[24]}$.

$$
k_{\beta}=\frac{1}{4}\left[k_{\beta}^{\|}+\left(k_{\beta}^{\| 2}+8 k_{\beta}^{\|} k_{\beta}^{\perp}\right)^{1 / 2}\right]
$$

where $k_{\beta}^{\|}=f_{\alpha} k_{\alpha}+f_{\gamma} k_{\gamma}, k_{\beta}^{\perp}=\left(f_{\alpha} / k_{\alpha}+f_{\gamma} / k_{\gamma}\right)^{-1} . f_{\alpha}$ is the volume fraction of ferrite, $f_{\gamma}$ is the volume fraction of cementite.

The matrix of cast iron is ferrite, pearlite, or a mixture of ferrite and pearlite. In this study, the range of $k_{\mathrm{m}}$ is $25-40 \mathrm{~W} \cdot \mathrm{m}^{-1} \cdot \mathrm{K}^{-1}$. The thermal conductivity of graphite is strongly anisotropic, different kinds of graphite have an extraordinary wide span ${ }^{[32]}$. Graphite of GCI is crystallized from melt, its lattice perfection 
is much better than that of ordinary polycrystalline graphite ${ }^{[2]}$. Therefore, for the thermal conductivity of graphite in GCI, we take the value of thermal conductivity of pyrolytic graphite with $1,950 \mathrm{~W} \cdot \mathrm{m}^{-1} \cdot \mathrm{K}^{-1}$ of $k_{\mathrm{a}}$ and $5.7 \mathrm{~W} \cdot \mathrm{m}^{-1} \cdot \mathrm{K}^{-1}$ of $k_{\mathrm{c}}$.

\subsection{Influence factors on thermal conductivity}

For GCI, the graphite volume fraction $f$ is usually $5 \%-10 \%$, the aspect ratio $p$ is $0.04-0.14$, the thermal conductivity of matrix $k_{\mathrm{m}}$ is $25-40 \mathrm{~W} \cdot \mathrm{m}^{-1} \cdot \mathrm{K}^{-1}$. The effects of these three factors on the thermal conductivity of GCI are discussed below.

\subsubsection{Graphite volume fraction}

Figure 10 shows the relationship between the effective thermal conductivity of GCI and the volume fraction of graphite, where $p$ is 0.1 and $k_{\mathrm{m}}$ is $30 \mathrm{~W} \cdot \mathrm{m}^{-1} \cdot \mathrm{K}^{-1}$. It can be seen that with the increase of graphite volume fraction, the thermal conductivity of GCI increases. This result suggests that flake graphite will indeed improve the overall thermal conductivity. A higher graphite volume fraction is beneficial to thermal conductivity of GCI.

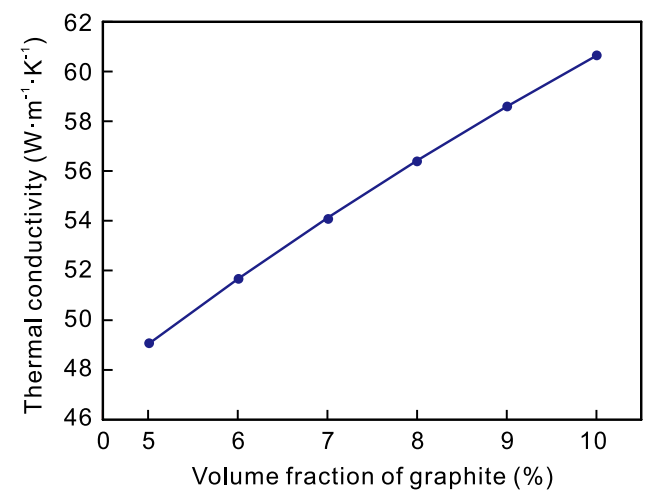

Fig. 10: Relationship between thermal conductivity of gray cast iron and graphite volume fraction, $p=0.1, k_{\mathrm{m}}=30 \mathrm{~W} \cdot \mathrm{m}^{-1} \cdot \mathrm{K}^{-1}$

\subsubsection{Aspect ratio of flake graphite}

Figure 11 shows the relationship between the effective thermal conductivity of GCI and the aspect ratio of flake graphite, where $f$ is $10 \%$ and $k_{\mathrm{m}}$ is $30 \mathrm{~W} \cdot \mathrm{m}^{-1} \cdot \mathrm{K}^{-1}$. With the increase of aspect ratio, the thermal conductivity of GCI decreases monotonously, which indicates that a lower aspect ratio of flake graphite is beneficial to obtaining high thermal conductivity of GCI. Of course, the aspect ratio of flake graphite is usually related to the size of eutectic cell. Therefore, with the increase of eutectic size, the aspect ratio of graphite decreases correspondingly, and the overall thermal conductivity of GCI will increase.

\subsubsection{Thermal conductivity of matrix}

Figure 12 shows the relationship between the effective thermal conductivity of GCI and the thermal conductivity of matrix, where $f$ is $10 \%$ and $p$ is 0.1 . With the increase of the matrix thermal conductivity, the thermal conductivity of GCI increases almost linearly. This result shows that the thermal conductivity of GCI depends not only on the flake graphite, but also on the matrix. According to Eq. (11), $k_{\alpha}$ mainly depends on the content of alloying elements. The lower the content

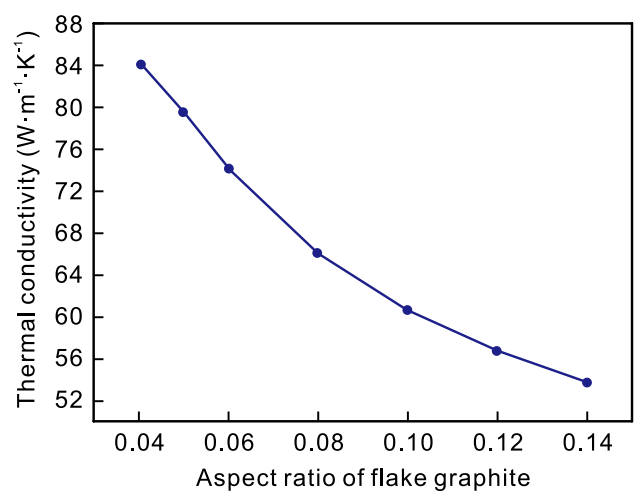

Fig. 11: Relationship between thermal conductivity of gray cast iron and aspect ratio of flake graphite, $f=10 \%, k_{\mathrm{m}}=30 \mathrm{~W} \cdot \mathrm{m}^{-1} \cdot \mathrm{K}^{-1}$

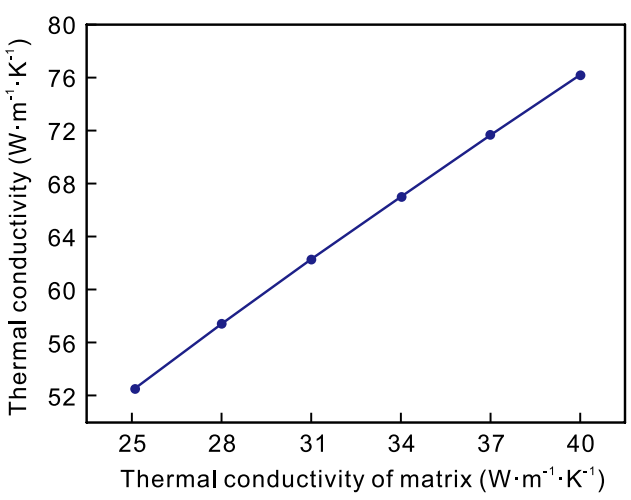

Fig. 12: Relationship between thermal conductivity of gray cast iron and matrix when $f=10 \%, p=0.1$

of alloying elements, the higher the thermal conductivity of ferrite. According to Eq. (12), the relationship between the content of alloying elements and the thermal conductivity of pearlite is similar to that of ferrite and the matrix of cast iron is ferrite, pearlite, or a mixture of ferrite and pearlite. Thus, the lower the content of alloying elements, the higher the thermal conductivity of matrix, and then the higher the overall thermal conductivity of GCI. For the GCI, the addition of alloying elements was always used to improve the mechanical properties. But in fact, the relationship between mechanical properties and thermal conductivity is often contradictory ${ }^{[2]}$. Therefore, the key method to design and develop high thermal conductivity GCI is controlling the alloying elements in GCI reasonably, especially controlling $\mathrm{Si}$ and $\mathrm{Al}$ elements.

\section{Conclusions}

A theoretical model of gray cast iron was designed taking into account a locally interconnected structure of flake graphite, and calculated the corresponding thermal conductivity by using the thermal resistance network method. Conclusions can be drawn as follows:

(1) The calculated results of this model are obviously higher than that of the effective medium approximation assuming that graphite is distributed in isolation, which indicates that the interconnected graphite structure significantly enhances the overall thermal conductivity. 
(2) High anisotropy of graphite thermal conductivity along the flake over perpendicular to the flake, high volume fraction of graphite, and small aspect ratio of flake graphite will make the connectivity effects of graphite more obvious to improve the overall thermal conductivity.

(3) Higher graphite volume fraction, lower aspect ratio and higher matrix thermal conductivity are beneficial to obtaining high thermal conductivity gray cast iron.

\section{Acknowledgments}

This work was supported by the National Natural Science Foundation of China (Grant No. 51371104).

\section{References}

[1] Riposan I, Chisamera M, Stan S. New developments in high quality grey cast irons. China Foundry, 2014, 11(4): 351-364.

[2] Wang G H, Li Y X. Thermal conductivity of cast iron - A review. China Foundry, 2020, 17(2): 85-95.

[3] Velichko A, Wiegmann A, Mücklich F. Estimation of the effective conductivities of complex cast iron microstructures using FIBtomographic analysis. Acta Materialia, 2009, 57(17): 5023-5035.

[4] Wang M, Pan N. Predictions of effective physical properties of complex multiphase materials. Materials Science and Engineering R: Reports, 2008, 63(1): 1-30.

[5] Gao B Z, Xu J Z, Peng J J, et al. Experimental and theoretical studies of effective thermal conductivity of composites made of silicone rubber and $\mathrm{Al}_{2} \mathrm{O}_{3}$ particles. Thermochimica Acta, 2015, 614: 1-8.

[6] Maxwell J C. A treatise on electricity and magnetism. Clarendon Press, 1881

[7] Eucken A. General regulations for the thermal conductivity of different types of substances and aggregate states. Research in the Field of Engineering A, 1940, 11(1): 6-20. (In German)

[8] Bruggeman V D A G. Calculation of various physics constants in heterogenous substances I Dielectricity constants and conductivity of mixed bodies from isotropic substances. Annals of Physics, 1935, 416(7): 636-664. (In German)

[9] Hamilton R L, Crosser O K. Thermal conductivity of heterogeneous two-component systems. Industrial \& Engineering Chemistry Fundamentals, 1962, 1(3): 187-191.

[10] Hatta H, Taya M, Kulacki F A, et al. Thermal diffusivities of composites with various types of filler. Journal of Composite Materials, 1992, 26(5): 612-625.

[11] Kapitza P L. Heat transfer and superfluidity of helium II. Physical Review, 1941, 60(4): 354.

[12] Hasselman D P H, Johnson L F. Effective thermal conductivity of composites with interfacial thermal barrier resistance. Journal of Composite Materials, 1987, 21(6): 508-515.

[13] Nan C W, Birringer R, Clarke D R, et al. Effective thermal conductivity of particulate composites with interfacial thermal resistance. Journal of Applied Physics, 1997, 81(10): 6692-6699.

[14] Faroughi S A, Huber C. Effective thermal conductivity of metal and non-metal particulate composites with interfacial thermal resistance at high volume fraction of nano to macro-sized spheres. Journal of Applied Physics, 2015, 117(5): 055104.

[15] Prieto R, Molina J M, Narciso J, et al. Thermal conductivity of graphite flakes-SiC particles/metal composites. Composites Part A: Applied Science and Manufacturing, 2011, 42(12): 1970-1977.
[16] Minnich A, Chen G. Modified effective medium formulation for the thermal conductivity of nanocomposites. Applied Physics Letters, 2007, 91(7): 073105.

[17] Xu J Z, Gao B Z, Kang F Y. A reconstruction of Maxwell model for effective thermal conductivity of composite materials. Applied Thermal Engineering, 2016, 102: 972-979.

[18] Marcos-Gomez D, Ching-Lloyd J, Elizalde M R, et al. Predicting the thermal conductivity of composite materials with imperfect interfaces. Composites Science and Technology, 2010, 70(16): 2276-2283.

[19] Bhuiyan M A, Pucha R V, Kalaitzidou K. 3D RVE models able to capture and quantify the dispersion, agglomeration, and orientation state of CNT in CNT/PP nanocomposites. Frontiers in Materials, 2016, 3: 1-13.

[20] Zehnder P, Schlunder E U. Thermal conductivity of granular materials at moderate temperatures. Chemie Ingenieur Technik, 1970, 42: 933-941.

[21] Hsu C T, Cheng P, Wong K W. A lumped-parameter model for stagnant thermal conductivity of spatially periodic porous media. ASME Journal of Heat Transfer, 1995, 117(2): 264-269.

[22] Yu B M, Li B W. Fractal-like tree networks reducing the thermal conductivity. Physical Review E, 2006, 73(6): 066302.

[23] Qian L J, Pang X M, Zhou J Q, et al. Theoretical model and finite element simulation on the effective thermal conductivity of particulate composite materials. Composites Part B: Engineering, 2017, 116: 291-297.

[24] Helsing J, Grimvall G. Thermal conductivity of cast iron: Models and analysis of experiments. Journal of Applied Physics, 1991, 70(3): 1198-1206.

[25] Holmgren D M, Diószegi A, Svensson I L. Effects of transition from lamellar to compacted graphite on thermal conductivity of cast iron. Cast Metals, 2006, 19(6): 303-313.

[26] Liu Y Z, Li Y F, Xing J D, et al. Effect of graphite morphology on the tensile strength and thermal conductivity of cast iron. Materials Characterization, 2018, 144: 155-165.

[27] Matsushita T, Saro A G, Elmquist L, et al. On the thermal conductivity of $\mathrm{CGI}$ and $\mathrm{SGI}$ cast irons. International Journal of Cast Metals Research, 2018, 31(3): 135-143.

[28] Zhai S P, Zhang P, Xian Y Q, et al. Effective thermal conductivity of polymer composites: theoretical models and simulation models. International Journal of Heat and Mass Transfer, 2018, 117: 358-374.

[29] Zhou S, Chiang S, Xu J, et al. Modeling the in-plane thermal conductivity of a graphite/polymer composite sheet with a very high content of natural flake graphite. Carbon, 2012, 50(14): 5052-5061.

[30] Nan C W. Physics of inhomogeneous inorganic materials. Progress in Materials Science, 1993, 37(1): 1-116.

[31] Fras E, Wiencek K, Górny M, et al. Graphite nodule and eutectic cell count in cast iron: theoretical model based on weibull statistics and experimental verification. Metallurgical and Materials Transactions, 2007, 38(2): 385.

[32] Balandin A A. Thermal properties of graphene and nanostructured carbon materials. Nature Materials, 2011, 10(8): 569.

[33] Ho C Y, Powell R W, Liley P E. Thermal conductivity of the elements. Journal of Physical and Chemical Reference Data, 1972, 1(2): 279-421.

[34] Wang G H, Li Y X. Effects of alloying elements and temperature on thermal conductivity of ferrite. Journal of Applied Physics, 2019, 126(12): 125118. 